\title{
The Nature of the Emission-line Nebulae in Powerful Far-infrared Galaxies
}

\author{
Lee Armus and Timothy M. Heckman \\ The Johns Hopkins University \\ George K. Miley \\ Sterrewacht Leiden
}

\begin{abstract}
We discuss our program of narrow-band $(\mathrm{H} \alpha+[\mathrm{NII}])$ imaging of a sample of 30 powerful farinfrared galaxies (FIRG's) chosen to have far-infrared spectral energy distributions similar to the prototype FIRG's Arp 220, NGC 3690, NGC 6240, and M82. The emission-line nebulae of these IR color-selected sample (ICSS) galaxies as a class are both impressively large (mean half light radius, $\mathrm{r} \sim 1.3 \mathrm{Kpc}$, and mean diameter, $\mathrm{D} \sim 16 \mathrm{Kpc}$ ) and luminous $\left(\mathrm{L}_{\mathrm{TOT}} \sim 10^{8} \mathrm{~L}_{\mathrm{e}}\right.$; uncorrected for internal extinction). The mean total $\mathrm{H} \alpha+[\mathrm{NII}]$ luminosity of the FIRG's is comparable to that found for pairs of optically selected interacting galaxies (Bushouse, Lamb, and Werner 1988), but is a factor of -5 greater than that of isolated spirals (Kennicutt and Kent 1983). Only $\sim 25 \%$ of the nearby $(z \leq 0.10)$ FIRG's have morphologies suggesting that large HII-regions contribut significantly to their emission-line appearance. The broad-band morphologies of our IR color-selected galaxies fall into three major categories. Nearly $75 \%$ are single galaxy systems, with the remaining FIRG's being either multiple nuclei systems, or members of interacting pairs. Since we see few $(10 \%)$ currently interacting FIRG's, yet many $(80 \%)$ with highly distorted continuum morphologies, our IR color criteria may be preferentially selecting galaxies that have undergone highly inelastic, rapidly merging interactions.
\end{abstract}

As a class, FIRG's have far-infrared luminosities, $\mathrm{L}_{\mathrm{FIR}}=10^{10}-10^{12} \mathrm{~L}_{\mathrm{o}}$, and far-infrared to blue luminosity ratios $L_{F I R} / L_{B}=10-100$. The far-infrared radiation from these galaxies is believed to be primarily generated by warm $(T-50 \mathrm{~K})$ dust. The optical spectra of the FIRG's are almost always dominated by emission-lines. The most prominent of these lines are $\mathrm{H} \alpha$, [NII] $\lambda \lambda 6548,6583 \AA$, [SII] $\lambda \lambda 6717,6731 \AA$, [OI] $\lambda 6300 \AA, \mathrm{H} \beta$, and [OIII] $\lambda \lambda 4959,5007 \AA$. The sizes, morphologies, and overall luminosities of the FIRG emission-line nebulae are an important probe of their interstellar media. As such, they shed valuable light on the nature of the energy source behind the enhanced activity that readily manifests itself in the far-infrared.

Prior to the present investigation, continuum subtracted narrow band images existed for only a handful of these powerful far-infrared galaxies. Nonetheless it was clear that their emission-line morphologies were nothing like the HII-region dominated disks of normal spiral galaxies. Studies of Arp 220 and NGC 6240 have revealed large scale (tens of Kpc) intricate emission-line nebulosities (Heckman, Armus, and Miley 1987). Narrow-band imaging of the closest FIRG, M82, has uncovered a complex filamentary nebula (McCarthy, Heckman, and van Breugel 1987).

In an attempt to determine whether such spectacular morphology is common among the FIRG's as a class, we have undertaken a narrow-band $(\mathrm{H} \alpha+[\mathrm{NII}])$ survey of 30 galaxies having 
spectral indices, $\alpha \leq-1.5$ betwee $25 \mu$ and $60 \mu$, and $\alpha \geq-0.5$ between $60 \mu$ and $100 \mu$ (where $S_{v} \propto$ $\left.v^{\alpha}\right)$. The 30 galaxies that make up this study span a large range in redshift $(z=0.001-0.169)$ and far-infrared luminosity $\left(\mathrm{L}_{\mathrm{FIR}}=10^{10}-10^{12} \mathrm{~L}_{\odot}\right)$.

The results of our narrow-band imaging study can be summarized as follows:

(1) - The ICSS FIRG's posess nebular morphologies that range from smooth and structureless to highly complex and chaotic (see Fig. 1), with some showing evidence for ordered structure on scales of a few Kpc to tens of Kpc. Of the 7 ICSS FIRG's that are apparently highly flattened in $\mathrm{R}(\mathrm{a} / \mathrm{b} \geq 2.0), 6$ have $\mathrm{H} \alpha+[\mathrm{NII}]$ isophotes that are either more circular than, or perpendicular to their continuum isophotes. This structure may represent emission-line gas that is extended out of the plane of the galaxy.

(2) - Overall the ICSS FIRG emission-line nebulae are quite large, having a mean diameter (maximum extent), $\mathrm{D} \sim 16 \mathrm{Kpc}$, and a mean half-light radius, $\mathrm{r}_{\mathrm{e}}-1.3 \mathrm{Kpc}$. Typically $2 / 3$ of the total $\mathrm{H} \alpha+[\mathrm{NII}]$ emission originates from beyond a radius of $1 \mathrm{Kpc}$.

(3) - The mean total $\mathrm{H} \alpha+[\mathrm{NII}]$ luminosity of the nebulae is $-5 \times 10^{41} \mathrm{erg} \mathrm{s}^{-1}$ (uncorrected for internal extinction). This is comparable to that found for systems (pairs) of optically selected interacting galaxies (Bushouse, Lamb, and Werner 1988), but is a factor of -5 greater than that found for isolated spirals (Kennicutt and Kent 1983; see Fig. 2). Typical ICSS FIRG's however, may have intrinsic $\mathrm{H} \alpha+[\mathrm{NII}]$ luminosities that are up to 30 times greater than average noninteracting spiral galaxies, and up to 6 times greater than the most luminous known noninteracting spiral galaxies (Kennicutt 1983). Only $-25 \%$ of the nearby $(z \leq 0.10)$ ICSS FIRG's have morphologies suggesting that large HII-regions contribute significantly to their emissionline appearance. While previous studies indicate that young stars dominate the optical spectra of the ICSS FIRG's (Armus, Heckman, and Miley 1989), a majority of the nebulae are evidently not powered by hot, young stars distributed in the same manner as they are in normal spiral galaxies (within giant $\mathrm{OB}$ associations).

(4) - The $\mathrm{H} \alpha+[\mathrm{NII}]$ and the far-infrared luminosities are correlated with the R-band luminosity, with both $\propto \mathrm{L}_{\mathrm{R}}{ }^{1.5-1.7}$ (see Fig. 3).

Nearly $75 \%$ of the ICSS FIRG's are single galaxy systems, with the remaining galaxies being either multiple nuclei systems, or members of interacting pairs. Since only $\sim 10 \%$ are currently interacting, yet nearly $80 \%$ have distorted continuum morphologies, our IR color criteria may be preferentially selecting galaxies undergoing highly inelastic, rapidly merging interactions. A small, but non-neglegible fraction ( $20 \%)$ of all the ICSS FIRG's appear completely undisturbed morphologically. These morphologically quiescent ICSS FIRG's have far-infrared luminosities that are comparable to those found for the sample as a whole.

\section{Conclusions}

The morphologies of the ICSS FIRG nebulae are very different from those of normal star- 
forming disk galaxies, yet young stars apparently dominate the optical continua (Armus, Heckman, and Miley. 1989). We therefore propose that a FIRG be visualized as a galaxy-scale "super-giant" HII-region: a galaxy whose large-scale ISM is being ionized by UV radiation and possibly mass outflows produced by an intense circumnuclear burst of star formation. In the vast majority of FIRG's this enhanced star formation has apparently been triggered by a galactic interaction. The fact that we see few currently interacting sample galaxies suggests that FIRG's may be the victims of rapidly merging encounters.

\section{References}

Armus, L., Heckman, T.M., and Miley, G.K. 1989, Ap.J., 347, 747.

Bushouse, H.A., Lamb, S.A., and Werner, M.W. 1988, Ap.J., 335, 74.

Heckman, T.M., Armus, L., and Miley, G.K., 1987, A.J., 93, 276.

Kennicutt, R.C. 1983, Ap.J., 272, 54.

Kennicutt, R.C., and Kent, S.M. 1983, A.J., 88, 1094.

McCarthy, P.J., Heckman, T.M., and vanBruegel, W. 1987, A.J., 93, 264.

\section{Figure Captions}

Fig. 1 - Emission-line $(\mathrm{H} \alpha+[\mathrm{NII}])$ and continuum (R-band) contour plots of select FIRG's. The lowest level is $10^{-16} \mathrm{erg} \mathrm{cm}^{-2} \mathrm{~s}^{-1} \operatorname{arcsec}^{-2}$ (in the galaxy rest frame) in the $\mathrm{H} \alpha+[\mathrm{NII}]$ contours, and $4 \mathrm{x}$ rms in the sky for the R-band contours. All levels are a factor of 2 higher in flux above the previous level. For each galaxy the $\mathrm{H} \alpha+[\mathrm{NII}]$ contour is on the left and the Rband contour is on the right. In all cases North is towards the top, East is towards the left, and a projected angular size of 10 arcseconds is indicated by a bar.

Fig. 2 - Plot of the far-infrared luminosity $\left(\mathrm{L}_{\mathrm{FIR}}\right)$ against the total $\mathrm{H} \alpha+[\mathrm{NII}]$ luminosity $\left(\mathrm{L}_{\mathrm{TOT}}\right)$ for the sample FIRG's. Also plotted are the loci of interacting spiral galaxies taken from Bushouse, Lamb, and Werner (1988), and the loci of isolated spiral galaxies taken from Kennicutt and Kent (1983). The solid diagonal lines represent select ratios of $\mathrm{H} \alpha+[\mathrm{NII}]$ to far-infared luminosity. Note that the $\mathrm{L}_{\mathrm{TOT}}$ and $\mathrm{L}_{\mathrm{FIR}}$ for the interacting galaxies of Bushouse, Lamb, and Werner (1988) represent $1 / 2$ of the total system $\mathrm{H} \alpha+$ [NII] and far-infrared luminosities, respectively.

Fig. 3 - Plot of (a) the total $\mathrm{H} \alpha+$ [NII] luminosity against the total absolute R-band magnitude, and (b) the far-infrared luminosity against the total absolute R-band magnitude for the sample FIRG's. The linear least-squares fit to the data is drawn as a dotted line in Figs. $3 a$ and $3 b$. 


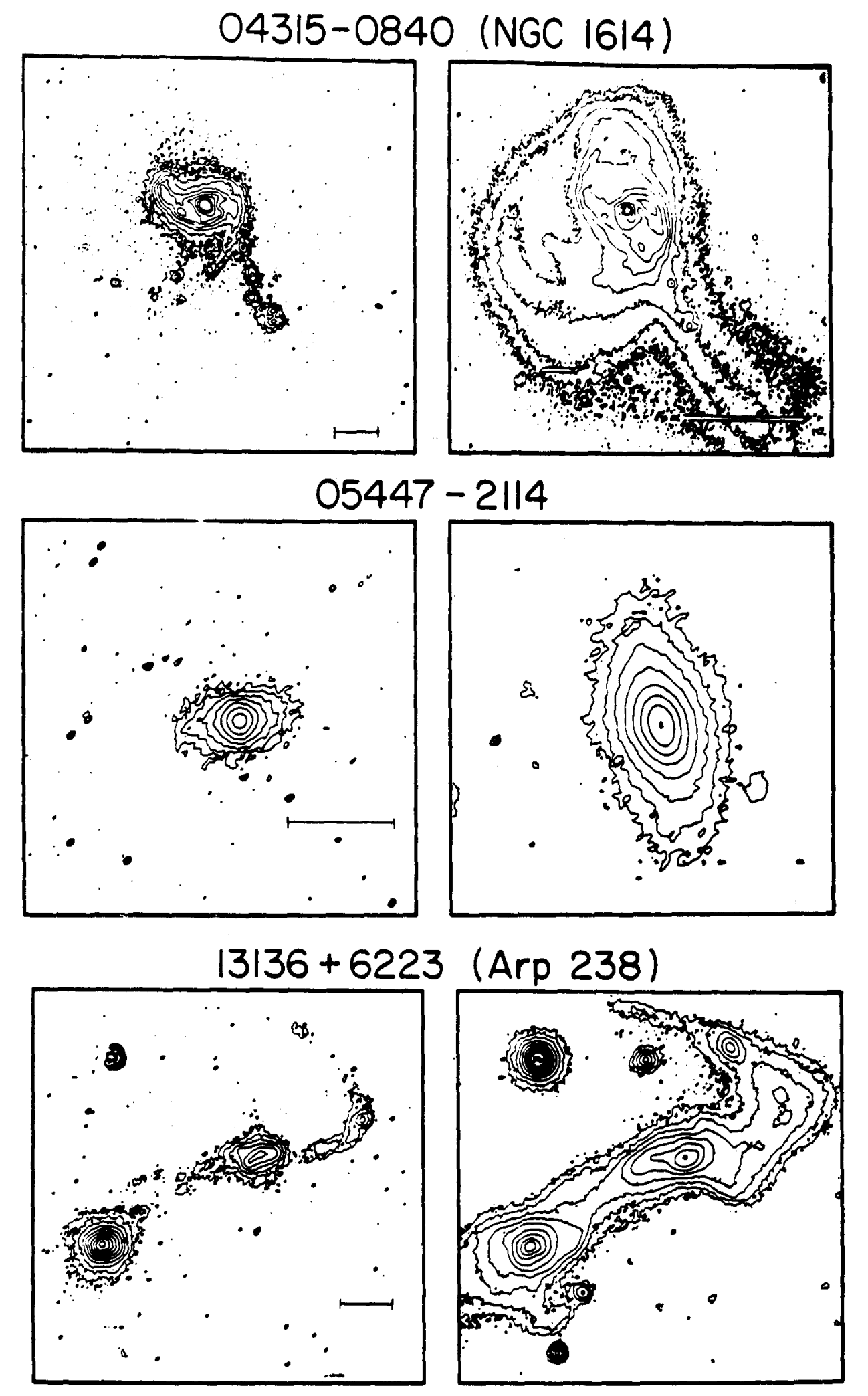

Fig. 1 


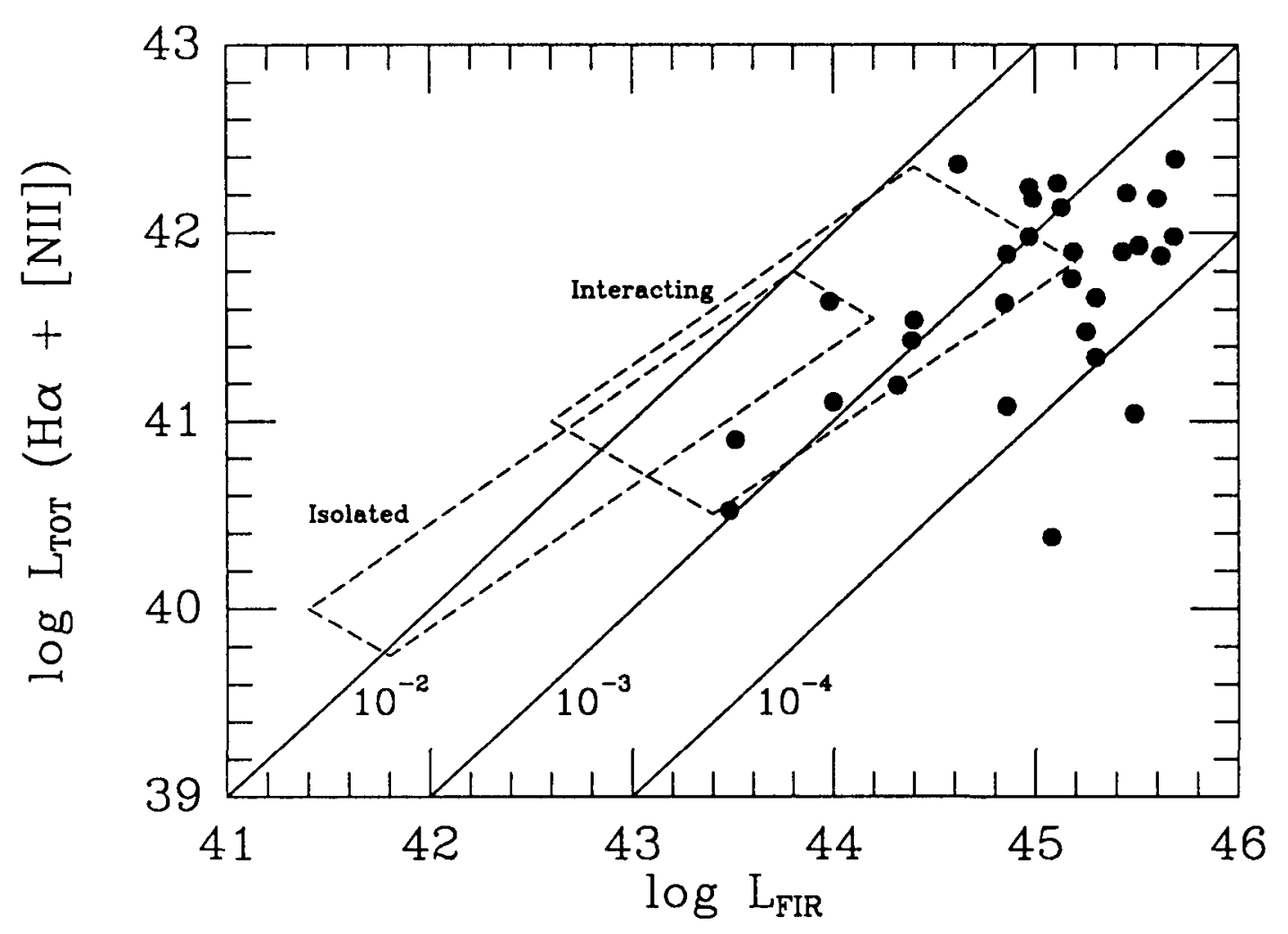

Fig. 2
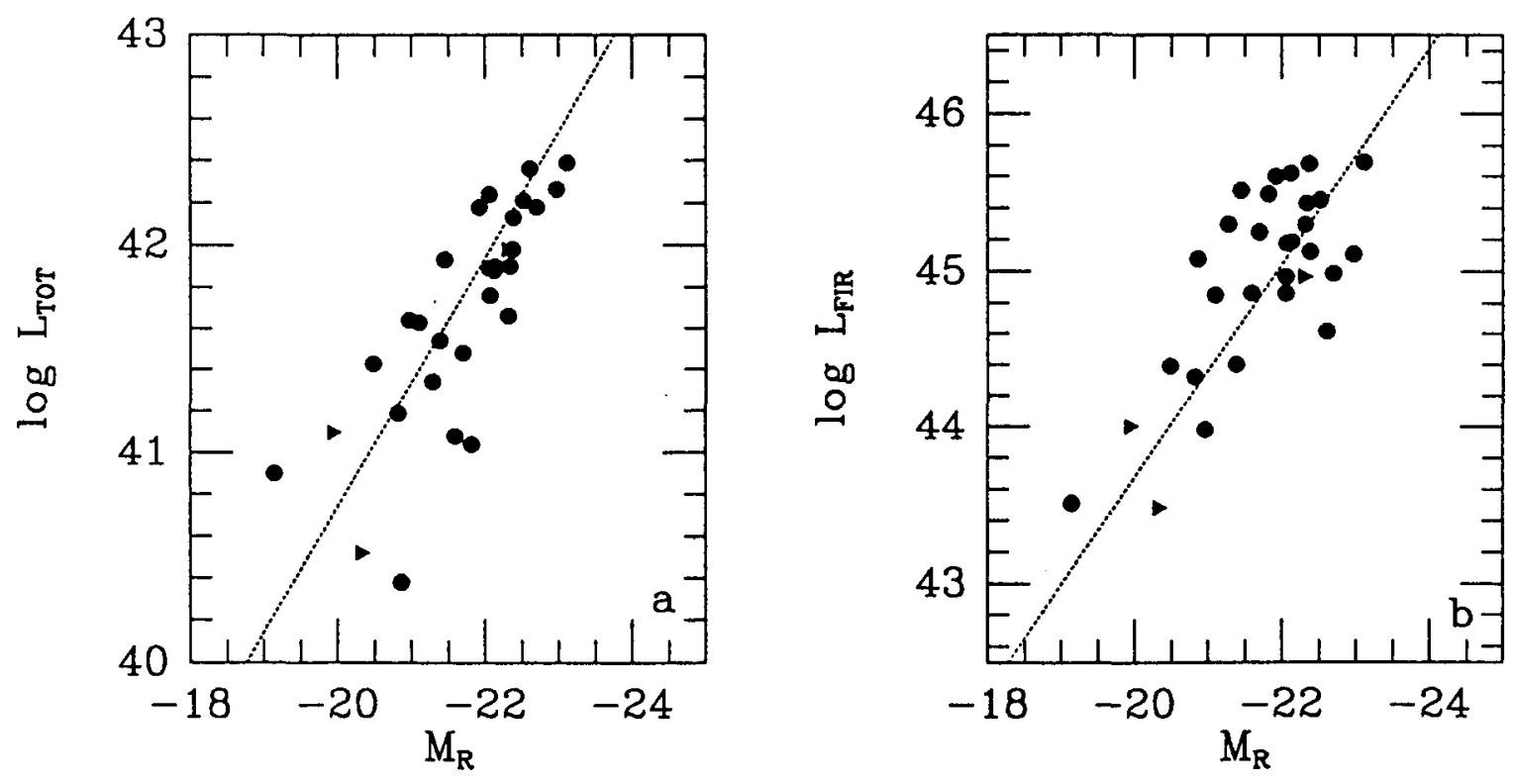

Fig. 3 